Classification

Physics Abstracts

$61.16 \mathrm{D}-61.70 \mathrm{~J}-81.40 \mathrm{G}$

\title{
A newly developed high resolution hot stage and its application to materials characterization
}

\author{
T. Kamino and H. Saka $\left(^{\star}\right)$ \\ Techno Research Laboratory, Hitachi Instrument Engineering Co. Ltd., 882, Ichige, Katsuta, Ibaraki \\ 312, Japan
}

(Received June 13, 1993; accepted March 8, 1993)

\begin{abstract}
This paper describes the newly developed specimen hot stage and its applications. The hot stage is designed for high resolution TEM image observation at high temperatures and EDX analysis at room temperature. A fine metal wire is employed as the heating element of the stage and battery is used as the heating power source. The hot stage is particularly useful for particle specimens. By using this novel stage, a HREM observation of Si at high temperatures $\left(\sim 1400^{\circ} \mathrm{C}\right)$ has been carried out. A dynamical observation of the surface of a $\mathrm{PbTiO}_{3}$ particle during heating and its chemical analysis using EDX before and after heating are also carried out.
\end{abstract}

\section{Introduction.}

Many kinds of hot stages have been developed in the past, and used for in-situ experiments in TEM [1-5]. There are two types of specimen heating methods. One is direct heating and the other is indirect heating method. The hot stages of earlier time employed the direct heating method using the grid or ribbon of metal as a heating element. This type of hot stages enables one to obtain high temperature with relatively low power input and the temperature of the specimen is possible to control directly and quickly. However, with this type of stage, the displacement of the specimen during heating is very large because of a relatively large thermal mass of the heating element. Thus, to obtain high resolution TEM image with hot stage of directly heating method is difficult and it was the main reason why this type of stage is not used in recent years. The present paper deals with a performances of a newly developed hot stage and its application to materials characterization. The hot stage has a fine metal wire as the heating element and battery as its power source.

Dynamical high resolution TEM image observation and its recording with normal TEM film at high temperatures and EDX analysis of the identical area at room temperatures are the two main features of the stage. A study of high resolution transmission electron microscopy of heated single crystal Si particles, and a study of structural and compositional change of a $\mathrm{PbTiO}_{3}$ to $\mathrm{Ti} / \mathrm{TiO}$ by the heating has been carried out using the hot stage.

$\left(^{\star}\right)$ Department of Engineering, Nagoya University, Chikusa-ku, Nagoya 466 Japan. 


\section{Instrument.}

A schematic diagram of the system of the hot stage is shown in figure 1 . The stage is designed for use with a side entry goniometer. The specimen heating current is controlled by a controller and measured by an ampere meter. Battery is used as a power source of the heater. It provides the current without any instability or fluctuation which affect the TEM image quality. A fine wire (diameter: $20 \sim 30 \mu \mathrm{m}$ ) of refractory metals such as $W$ is used as a heating element (Fig. 2). Figure 3 is an example of the heating temperature calibration for a heating element of a tungusten wire with the diameter of $25 \mu \mathrm{m}$. The hot stage was brought into a vacuum evaporator and heated there. The measurement of the heating temperature was carried out by using a pyrometer from out side of the vacuum evaporator through bell through the bell jar. Specimens of particles were put on the wire heater directlyand only the steady particles on the wire were selected and examined. The stage with such a small thermal mass has quite important advantages for in-situ experiments. First, drift of the specimen during heating is very small and it made a recording of high resolution TEM image with TEM film possible even at very high temperatures like over $1000^{\circ} \mathrm{C}$. Second, no large capacity heating power is required. Indeed, two UM-2(1.5V) batteries are enough to keep the heater of $25 \mu \mathrm{m}$ diameter $W$ at $1500{ }^{\circ} \mathrm{C}$ for about 10 hours. The hot stage can be designed in the thickness of less than $2 \mathrm{~mm}$ and use with high resolution objective lens pole-piece is possible. Since the physical volume of the stage is so small, the X-ray noise from the stage is so low as to make EDX analysis of a nano-meter area possible. Because the thermal mass of the heating element isalso so small, the specimen temperature can be controlled in short time whenever it is required. In other words, a high resolution TEM image recording at high temperature and EDX analysis at low temperature are possible to carry on alternately through a series of experiment.

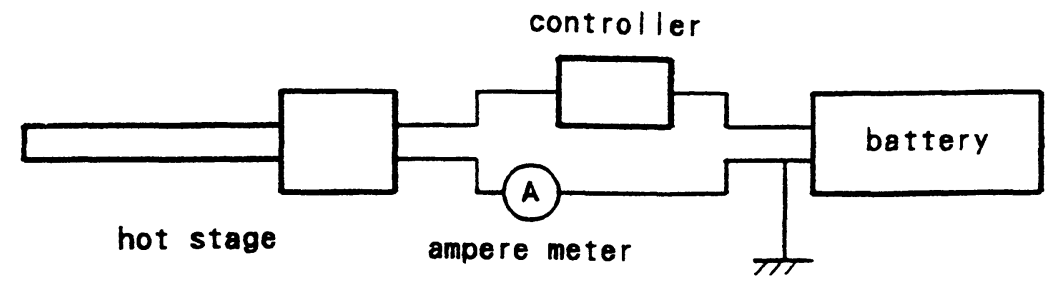

Fig. 1. - Schematic diagram of the specimen hot stage.

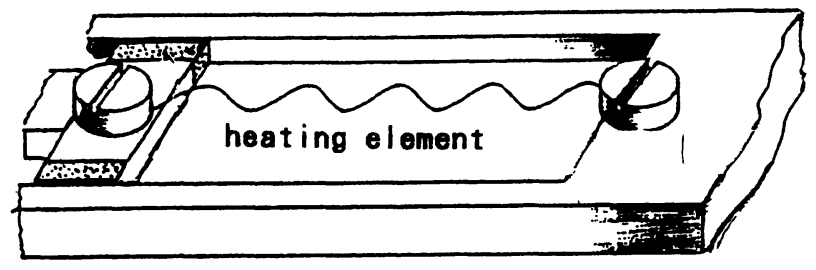

Fig. 2. - An example of wiring of the heating element. 


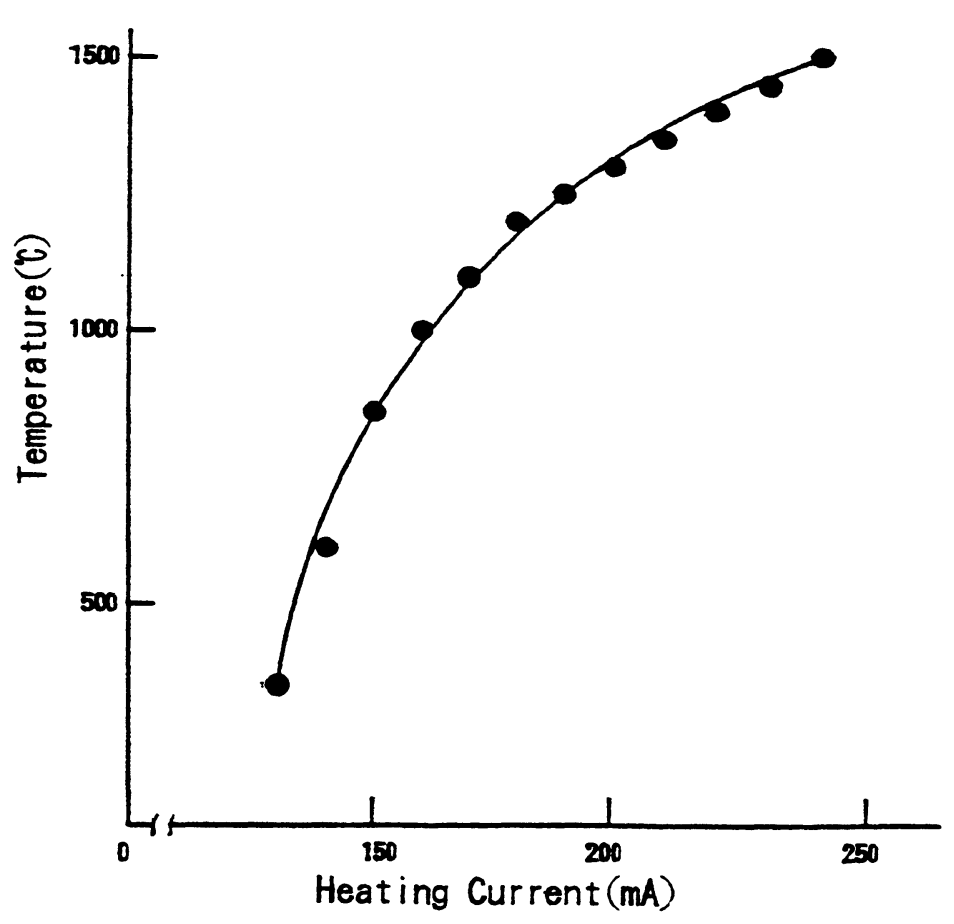

Fig. 3. - The calibration curve of the heating temperature for $25 \mu \mathrm{m}$ diameter $W$ wire.

\section{Applications.}

The microscope used for the experiment is a Hitachi H-9000NAR 300kV high resolution analytical TEM equipped with a Kevex Delta EDX system and a Gatan 622 high resolution TV system. The detector of the EDX system has ultra thin window (UTW) and its take-off angle and solid angle are $22^{\circ}$ and $0.12 \mathrm{sr}$, respectively. The spherical and chromatic aberration coefficients of the objective lens are $0.69 \mathrm{~mm}$ and $1.4 \mathrm{~mm}$, respectively. Point-to-point resolution of the microscope is $0.175 \mathrm{~nm}$. The maximum specimen tilting angle of the stage is $\pm 15^{\circ}$ when the hot stage is in use.

3.1 HREM STUDY OF A HEATED Si PARTICLE. - By using the newly developed hot stage, a fine structure of $\mathrm{Si}$ at high temperature has been studied. Figure 4a shows a TEM image of single crystalline Si particles before heating. Figure $4 \mathrm{~b}$ shows a TEM image of the same particle after increasing the heater temperature to $1400^{\circ} \mathrm{C}$. By the heating, the particles has changed its shape remarkably. The two particles $\mathrm{A}$ and $\mathrm{B}$ coalesced into one. These phenomena may be regarded as indicating that actual specimen temperature was quite close to the melting point of $\mathrm{Si}\left(1414^{\circ} \mathrm{C}\right)$. Indeed, when the temperature of the heating element was increased to as high as $1500{ }^{\circ} \mathrm{C}$, the $\mathrm{Si}$ particles got molten completely (Fig. 4c).

A high magnification TEM image and the corresponding electron diffraction pattern of the crystal at about $1400{ }^{\circ} \mathrm{C}$ are shown in figure 5. The image has been recorded on normal EM photographic film with an exposure time of 2 seconds. The orientation of the particle was aligned in the $\{110\}$-zone-axis by means of the tilting facility of the specimen stage. The structure images (within circle) together with lattice fringes of (111) $d=0.314 \mathrm{~nm}),(220)(d=0.192 \mathrm{~nm})$ and 


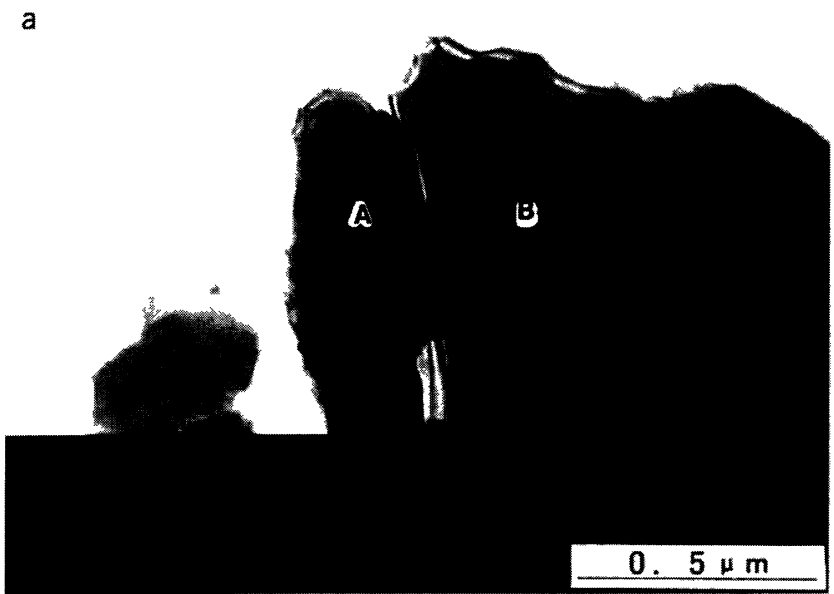

b
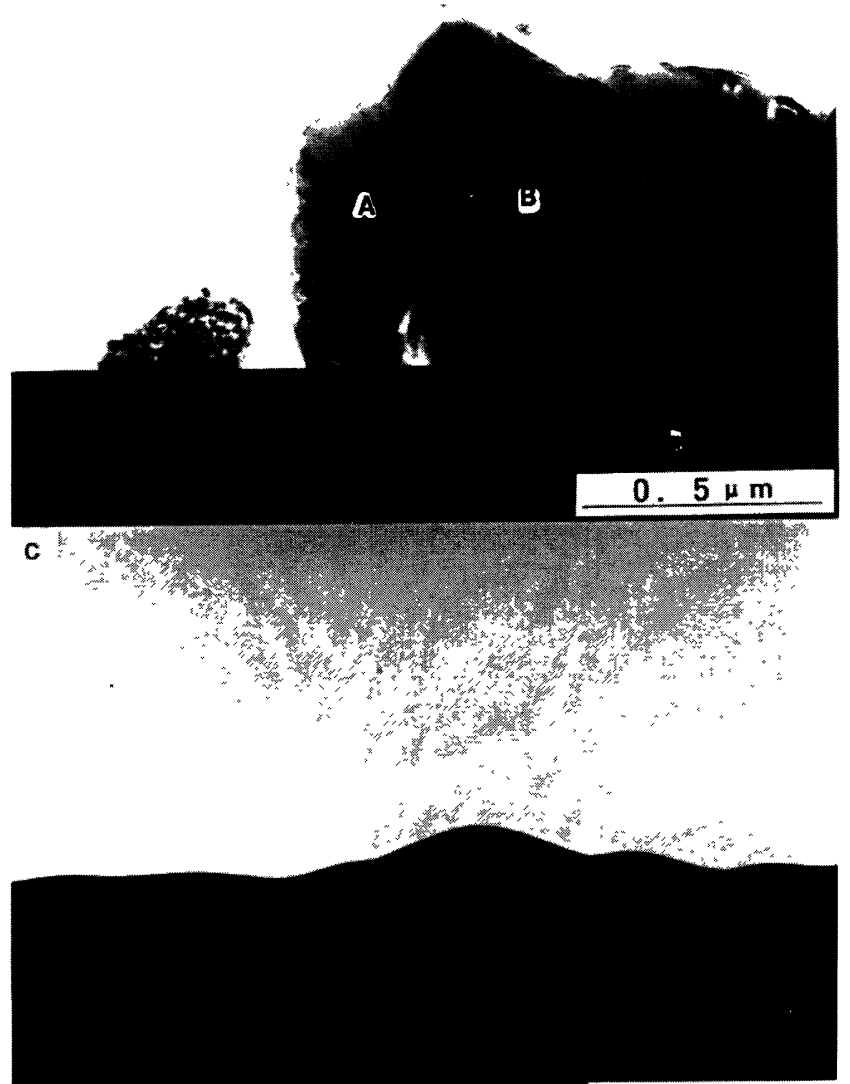

\section{$0.5 \mu \mathrm{m}$}

Fig. 4. - TEM images of single crystal Si particles before heating(a), at $1400^{\circ} \mathrm{C}(\mathrm{b})$ and at $1500^{\circ} \mathrm{C}(\mathrm{c})$.

(400) $(d=0.136 \mathrm{~nm})$ are clearly resolved. Although the inside structure is so stable as can be seen in the picture, the surface is quite unstable. Figure $6 a$ and $b$ are the images recorded in 
about 5 seconds interval. A sub-grain boundary changes its shape and dislocations in the grain change its positions. The sharpness of the lattice fringes are partially poor. It is because atoms of the region moved during photographing.

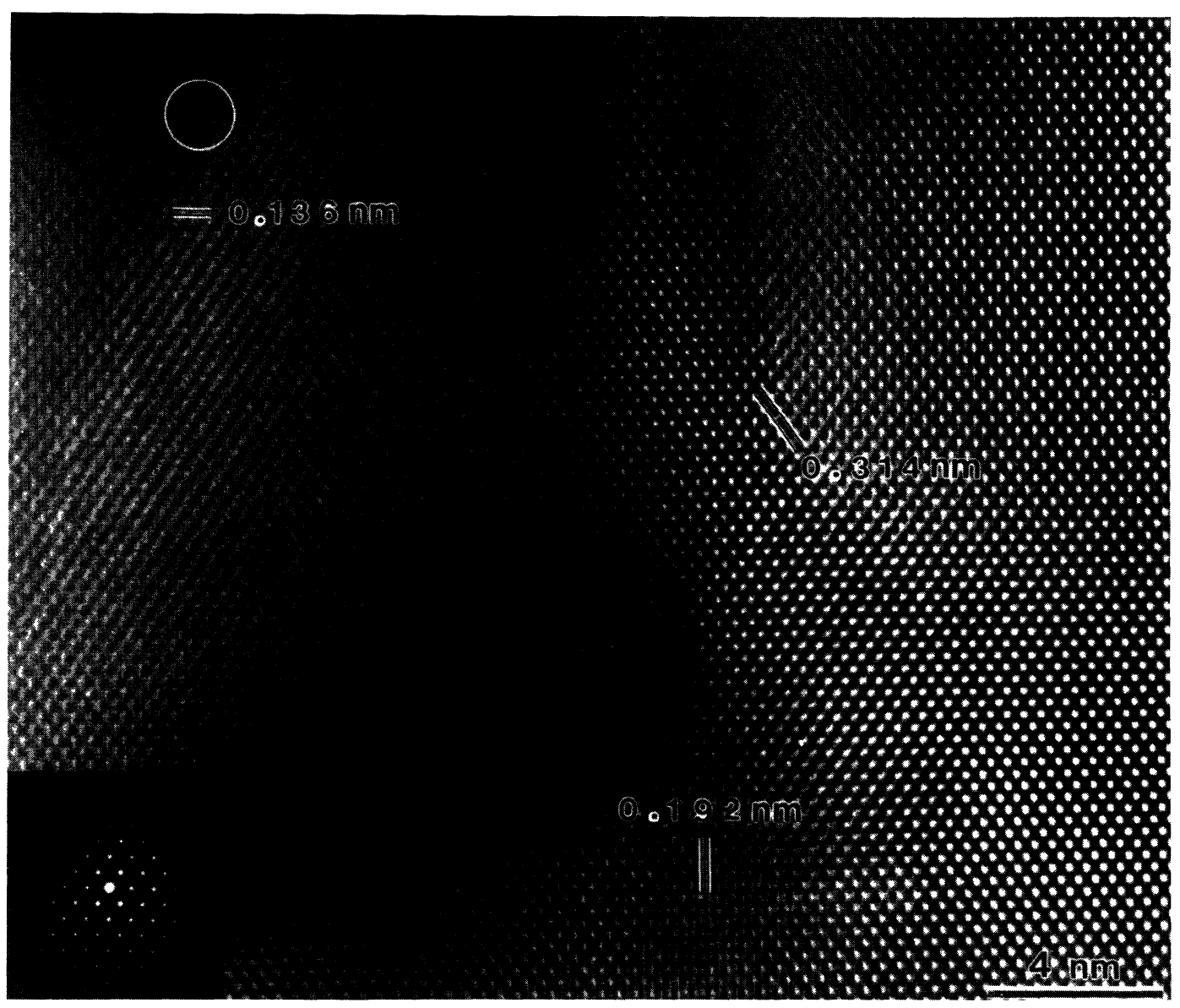

Fig. 5. - A HREM image and the corresponding electron diffraction pattern of the crystal shown in the center of figure $4 \mathrm{~b}$.

3.2 Structural AND COMPOSitional study of A $\mathrm{PbTiO}_{3}$ A PbTiO duRing heating. Figure 7 shows TEM images and electron diffraction patterns of a particle of $\mathrm{PbTiO}_{3}$ taken at room temperature, $320^{\circ} \mathrm{C}, 450{ }^{\circ} \mathrm{C}$ and $560^{\circ} \mathrm{C}$. This serial observation reveals that the initially amorphous $\mathrm{PbTiO}_{3}$ particle crystalized during the heating. Crystalization was completed at $560^{\circ} \mathrm{C}$, as can be seen in the diffraction patterns. Figure 8 shows the results of EDX analysis of the particle before and after heating. The result of EDX analysis indicates that $\mathrm{Pb}$ is not contained in the particle after heating. From both electron diffraction patterns and EDX analyses, it is confirmed that the initially amorphous $\mathrm{PbTiO}_{3}$ particle had transformed into a mixiture of $\mathrm{Ti}$ and TiO. That is, $\mathrm{Pb}$, initially contained in the particle, must have evaporated during the heating. 


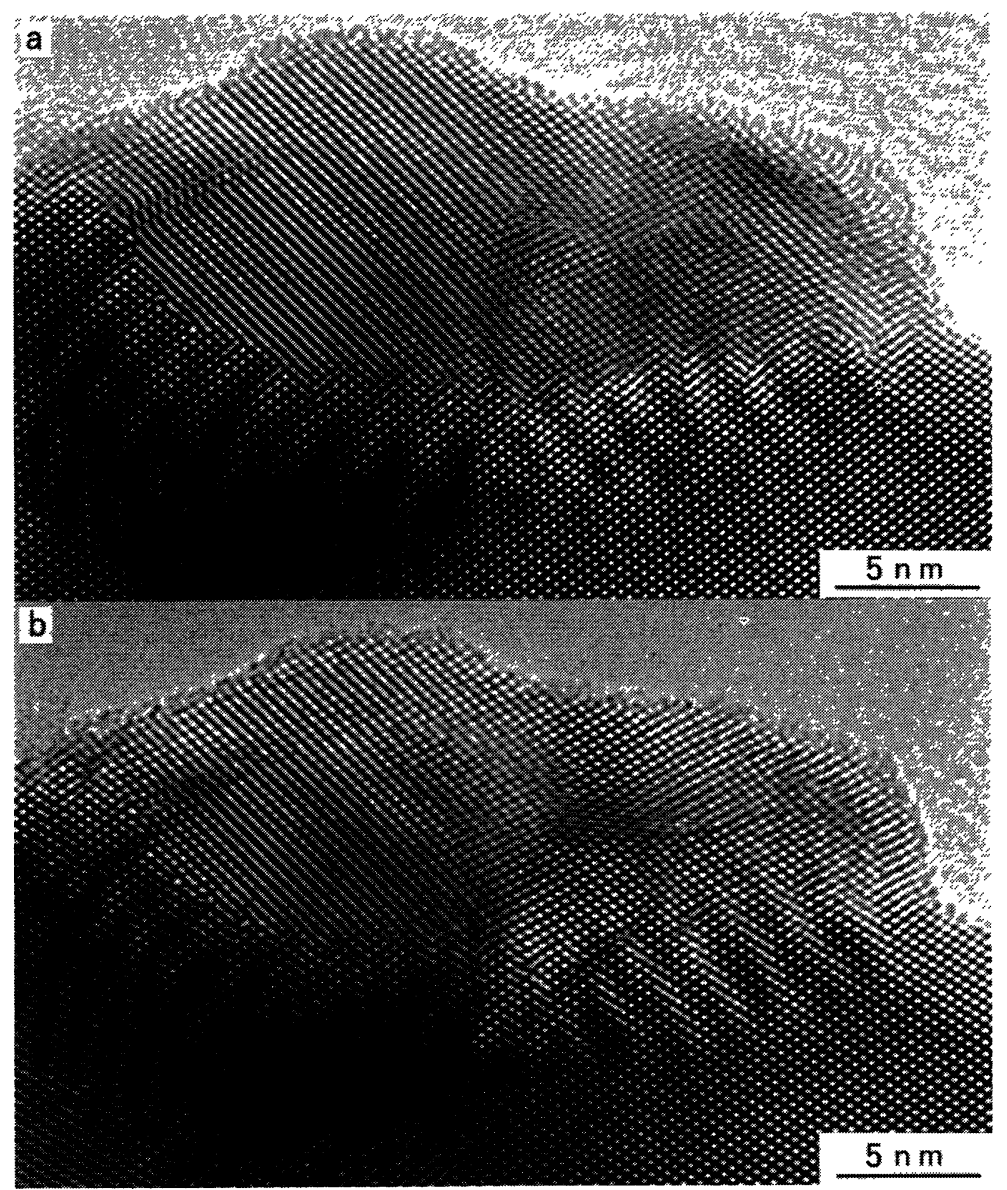

Fig. 6. - a) A high resolution TEM image of a surface Si particle at $1400^{\circ}$ C. b) Same area, 5 seconds later.

Figure 9 reproduces dynamical high resolution TEM images showing the structural change of the surface of the particle. Images were recorded at about $560{ }^{\circ} \mathrm{C}$. The dark layers appeared just beneath the surface of the particle and then left the surface layer by layer. This behavior, confirmed with the result of the EDX analysis of the particle after heating, suggested that the layers may be associated with the evaporation of $\mathrm{Pb}$.

\section{Conclusion.}

Since the hot stage used in the experiment was single tilt type, there was a difficulty in finding a specimen lying in proper orientation. There is a limitation in the size of a specimen because specimen of particle must be put on a fine wire directly without supporting substrate. However, as shown in the results of this paper, the hot stage of the direct heating type has excellent features for use with a high resolution analytical TEM. It allows an in situ high resolution TEM study at high temperatures and compositional analysis using EDX system. Use of a dry battery cell as the power source was quite effective in improvement of a high resolution TEM image observation. It 


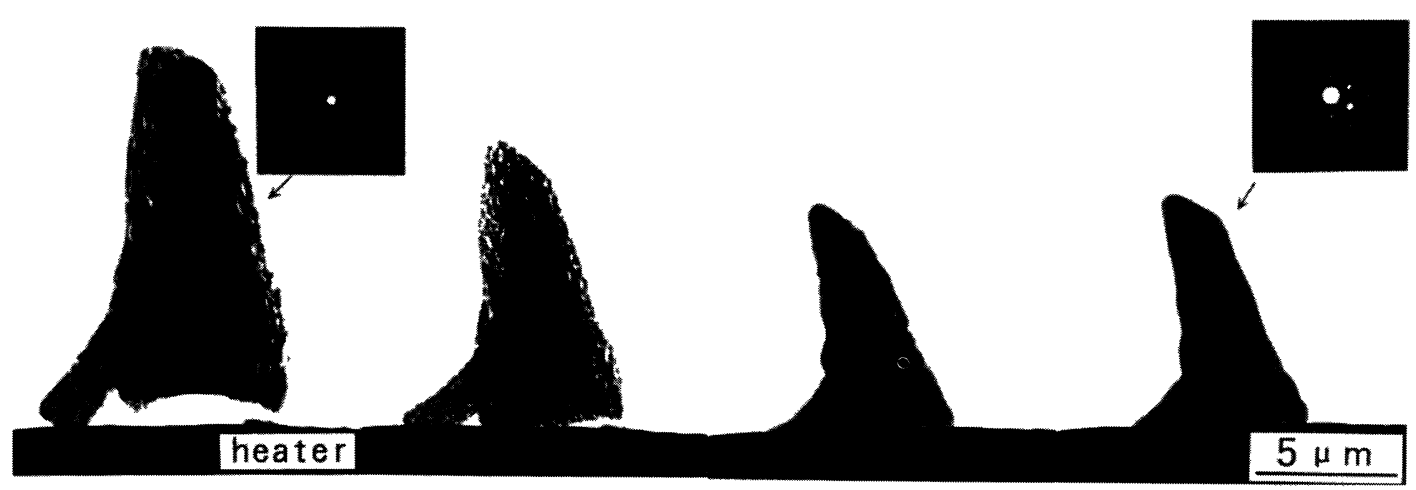

Fig. 7. - TEM images and electron diffraction patterns of the particle before heating, $320^{\circ} \mathrm{C}, 450{ }^{\circ} \mathrm{C}$ and $560{ }^{\circ} \mathrm{C}$ (left to right).

\section{before heating}
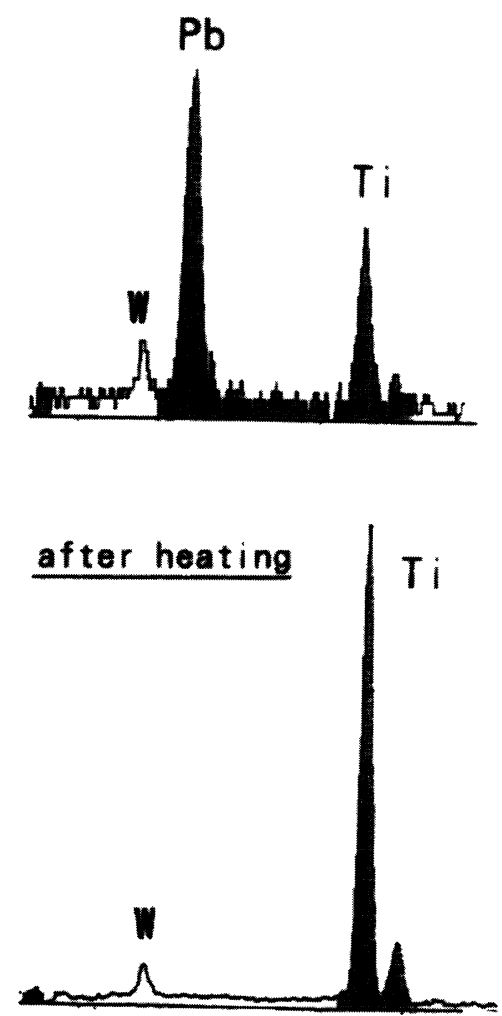

Fig. 8. - EDX analysis of the particle before and after heating ( $W$ : from the heater).

was also confirmed that there is no large temperature difference between heater and specimen. Another advantageous feature is simple design of heating components. It allows us to change 

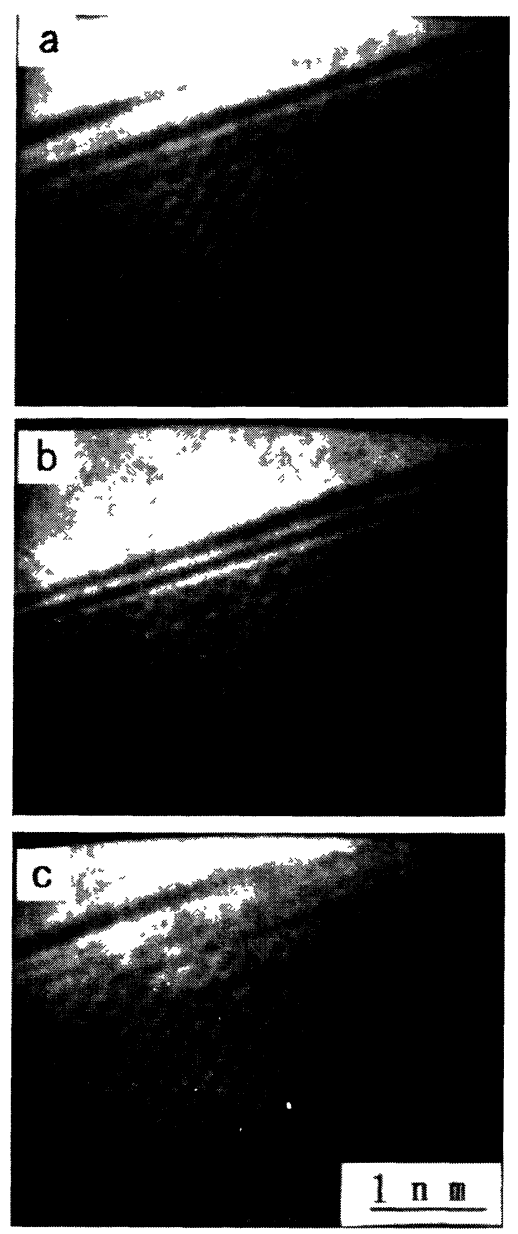

Fig. 9. - Reproduce of the dynamical high resolution observation of the surface of the particle.

a heating element quite easily. It means that the appropriate heating element can be chosen for each experimental purpose so that any artefacts due to the reaction between specimen and heating element can be minimized. By using the newly developed hot stage, a high resolution TEM image observation at high temperature and analysis of the identical micro area of the materials has become possible on near atomic scale.

\section{Acknowledgements.}

The author is grateful to Mr. M. Tomita of Instrument Division, Hitachi Ltd. for his support in manufacturing the hot stage used in this experiment. 


\section{References}

[1] Agar A.W. and LuCAS J.H., Proc. 5th Int. Congr. Electron Microsc., Philadelphia 1, E-2 (1962).

[2] WALLENBERGER R., BOVIN J.-O. and SMITH D.J., Naturwissenshaften 72 (1985) 139.

[3] Swann P.R., Proc. 5th Eur. Reg. Conf. Electron Microsc., Manchester (1972) p.330.

[4] SWANN P.R., Proc. 9th Int. Congr. Electron Microsc., Toronto 3 (1978) 319.

[5] MacPartland J.O.P., Proc. 5th Int. Congr. Electron Microsc., Philadelphia 1 E-3 (1962). 